\title{
Ambientes Físico-Virtuais de Aprendizagem
}

\author{
Rafael A. P. dos Santos ${ }^{12}$, Silvia S. C. Botelho ${ }^{12}$, Marcos A. A. Bichet ${ }^{1}$ \\ ${ }^{1}$ Centro de Ciências Computacionais - Universidade Federal do Rio Grande (FURG) \\ 96.203-900 - Rio Grande - RS - Brazil \\ ${ }^{2}$ Programa de Pós-Graduação em Educação em Ciências - Universidade Federal do Rio \\ Grande (FURG) \\ 96.203-900 - Rio Grande - RS - Brazil \\ \{rafaelpenna, silviacb\}@furg.br, marcosamaralrg@gmail.com
}

\begin{abstract}
Virtual Learning Environments are computer systems available on the internet, for the support of activities mediated by information and communication technologies. This paper focuses on the integration of real/physical elements in such environments, through advanced humancomputer interfaces. We propose the definition of Cyber-Physical Learning Environments, discussing their characteristics and a reference conceptual model. Finally, the Toogle plataform, proposed to implement cyber-physical systems, is used to develop these new spaces.
\end{abstract}

Resumo. Ambientes Virtuais de Aprendizagem são sistemas computacionais disponíveis na internet, destinados ao suporte de atividades mediadas pelas tecnologias de informação e comunicação. Este artigo tem como foco a integração de elementos reais/físicos em tais ambientes, através de interfaces humano-computador avançadas. Para tanto, propõe-se a definição de Ambientes Físico-Virtuais de Aprendizagem, discutindo suas características e um modelo conceitual de referência. Por fim a plataforma Toogle, proposta para implementação de sistemas físico-virtuais, é utilizada no desenvolvimento desses novos espaços.

\section{Introdução}

Recentemente, com o surgimento da internet, com o desenvolvimento da infraestrutura de banda larga, com e sem fio, e com o avanço no desenvolvimento de sistemas embarcados e mais rápidos, tem-se um novo cenário tecnológico mundial. O modo como as pessoas interagem com a tecnologia se modifica e começam a surgir as interfaces homem-computador avançadas, que facilitam a utilização dos computadores. Os ambientes passam a ter diversos dispositivos computacionais que influenciam de alguma maneira na vida das pessoas.

Nos últimos anos, estudos têm procurado unir dois mundos que até então existiam exclusivamente em separado. Propostas de Sistemas Físico-Virtuais (CyberPhysical Systems ou CPS) começam a aparecer, buscando integrar sistemas computacionais com objetos do mundo físico [Lee, 2008].

Este trabalho apresentará a plataforma Toogle, que foi desenvolvida para implementação de CPSs. A plataforma foi construída para permitir a criação e edição de ambientes que integrem os mundos físico e virtual, através de redes de sensores e atuadores, e também para propiciar a navegação do usuário por esses ambientes. 
Durante esse período de avanço tecnológico, tornou-se constante a discussão de como aproveitar essa gama de recursos que tem surgido, no contexto educacional. A partir do surgimento da educação à distância, aparecem os primeiros Ambientes Virtuais de Aprendizagem (AVA). Esses ambientes costumam oferecer uma série de objetos virtuais que buscam auxiliar a aprendizagem, como, por exemplo, imagens, vídeos, animações 2D e 3D, jogos, simulações, etc. Apresentam também ferramentas que propiciam comunicação entre estudantes e professores. Por outro lado, os AVAs não costumam utilizar objetos do mundo físico para fins de aprendizagem, ou seja, elementos que fazem parte do dia a dia do aluno, que podem apresentar alto grau de engajamento no ensino, não são explorados como potenciais instrumentos para aprendizagem.

São as questões relacionadas a integração dos ambientes físicos com os AVAs que passam a ser o foco deste trabalho, através da proposta de um Ambiente FísicoVirtual de Aprendizagem (AFVA), que será apresentado posteriormente. Acredita-se que o avanço tecnológico das últimas décadas tenha possibilitado a criação de interfaces humano-computador avançadas capazes de propiciar tal integração.

\section{Fundamentação Teórica e Trabalhos Relacionados}

Questões importantes para a utilização de AVAs e para o desenvolvimento de aplicações tecnológicas na educação, foram tomadas como eixos centrais para a análise a seguir apresentada. No final desta seção é apresentada uma tabela comparativa entre as principais referências analisadas.

\subsection{Ambientes Virtuais de Aprendizagem (AVAs)}

Segundo Sneha e Nagaraja, pode-se definir ambientes virtuais de aprendizagem como: "um sistema para transferência de materiais de aprendizagem para os alunos por meio da web. Estes sistemas constituem acompanhamento do estudante, ferramentas de comunicação, avaliação e colaboração...” [Sneha e Nagaraja, 2014].

Dillenbourg (2002) se propõe a identificar AVAs por uma série de características apresentadas a seguir: um AVA é um espaço para conceber informação; um AVA é um espaço social; o espaço virtual é explicitamente representado (podendo variar de texto a mundos imersivos 3D); os alunos não são apenas ativos, mas também atores; AVAs não se restringem a educação a distância; AVAs integram tecnologias heterogêneas e múltiplas propostas pedagógicas; a maioria dos ambientes virtuais acontecem simultaneamente aos ambientes físicos. A seguir apresenta-se, na Figura 1, o modelo conceitual de Dongming Xu (2005) para desenvolvimento de AVAs construtivistas. Posteriormente, na seção 3.2 propõe-se uma adaptação deste modelo conceitual, onde cada elemento é explicado. A seguir é apresentado um dos principais AVAs atuais, tomando como referência alguns eixos de análise.

\section{Moodle}

O moodle é um dos AVAs existentes atualmente mais populares devido a sua fácil utilização e sua modularidade (diversos plug-ins). Possui uma vasta variedade de opções de gerenciamento, como diferentes possibilidades de autenticação, de criação de perfis e de regras permissão para inscrição em cursos. Apresenta também diversas ferramentas de administração de cursos, de comunicação e de avaliação, como chat, forums, wikis, construtores de avaliação, entre outras. Possui versões para smartphones, o que de certa maneira contribui para um maior engajamento dos alunos. 


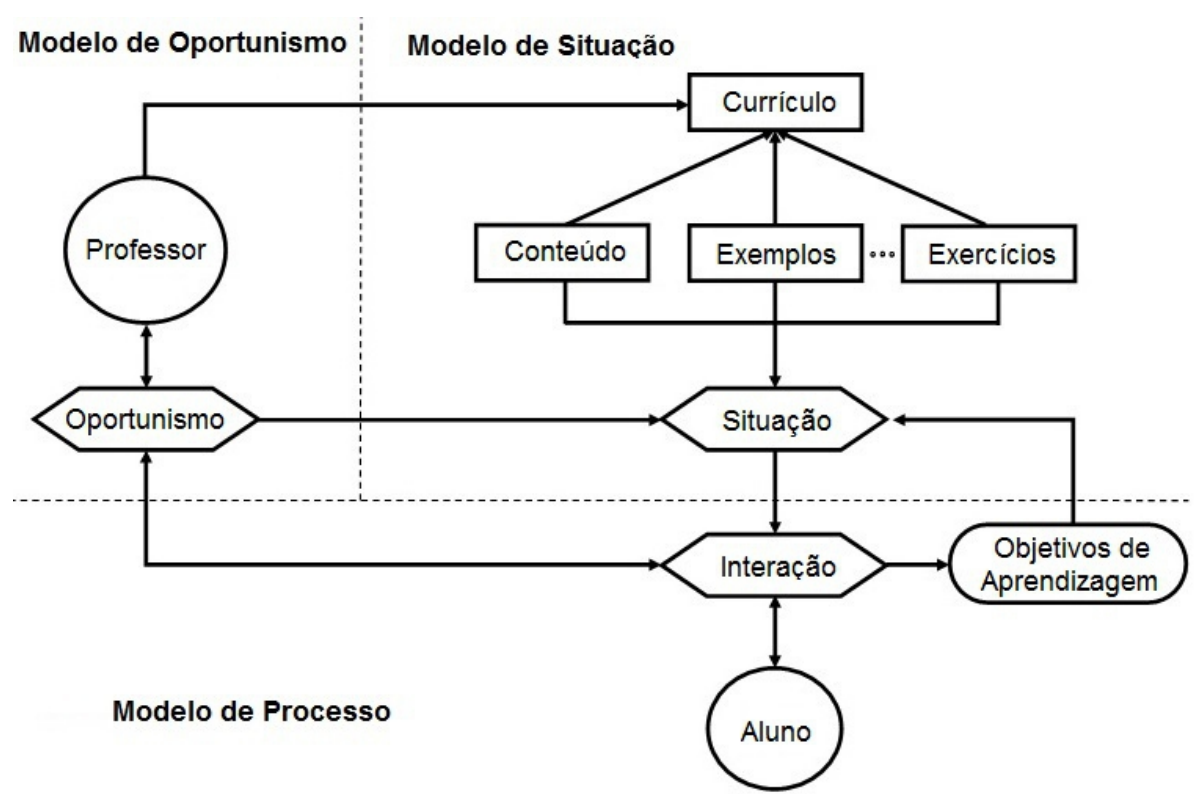

Figura 1: Modelo Conceitual para AVAs, tradução de (Dongming Xu, 2005)

\subsection{Interfaces Humano-Computador Avançadas}

A seguir, discute-se sobre CPSs, IHC avançada que possibilita interações diferenciadas. Na tabela comparativa do final da seção outras IHCs serão apresentadas também.

\section{CPS}

Sistemas Físico-Virtuais (Cyber-Physical Systems ou CPS) são integrações de sistemas computacionais com processos físicos [Lee, 2008]. Um CPS agrega recursos de computação, comunicação e armazenamento para monitorar e controlar entidades do mundo físico, de forma confiável, segura, eficiente e em tempo real [Sha, 2008].

Quanto a propostas educacionais que envolvem sistemas físico-virtuais, o trabalho de Olympiou e Zacharia (2012) tem como objetivo investigar o efeito de experiências com a compreensão de conceitos relacionados à luz e cores, através de objetos físicos e objetos virtuais. Embora a proposta de Olympiou e Zacharia (2012) envolva um alto grau de interação com objetos (físicos), as possibilidades de comunicação entre alunos e professores não parece ser explorada. Os autores estabelecem protocolos com questionários e procedimentos para a avaliação da aprendizagem dos alunos. O trabalho apresenta um bom engajamento, uma vez que propõe a utilização de elementos físicos, do mundo real dos alunos, além de virtuais. Também se destaca por possuir boa usabilidade e abordagem multissensorial, já que permite a manipulação de objetos físicos, trabalhando o tato, a visão, motricidade, etc.

A seguir é apresentada uma tabela comparativa entre as principais referências estudadas, a partir dos eixos centrais estabelecidos.

Pela tabela 1, observa-se que, em geral, os AVA possuem boas ferramentas administrativas, de avaliação e de comunicação. No entanto, as questões que envolvem o engajamento, a abordagem multissensorial e a utilização de objetos físicos no ensino, são pouco abordadas nesses ambientes. 
Tabela 1. Quadro comparativo

\begin{tabular}{|c|c|c|c|c|c|c|c|c|}
\hline $\begin{array}{l}\text { Referêncial } \\
\text { Critério }\end{array}$ & Comunicação & $\begin{array}{c}\text { Ferramentas } \\
\text { administrativas } \\
\text { e de avaliação }\end{array}$ & $\begin{array}{l}\text { Abordagem } \\
\text { pedagógica }\end{array}$ & \begin{tabular}{|c|} 
Grau de \\
colaboração \\
e \\
Ferramentas \\
de Autoria
\end{tabular} & $\begin{array}{c}\text { Grau de } \\
\text { "inteligência" }\end{array}$ & $\begin{array}{l}\text { Utilização } \\
\text { de } \\
\text { objetos } \\
\text { físicos/ } \\
\text { reais }\end{array}$ & $\begin{array}{c}\text { Engajamento } \\
\mathrm{e} \\
\text { Usabilidade }\end{array}$ & $\begin{array}{l}\text { Abordagem } \\
\text { Multi- } \\
\text { sensorial }\end{array}$ \\
\hline WebCT & $X X$ & $\mathrm{XXX}$ & $X X$ & $X X$ & - & - & $x$ & $X$ \\
\hline Moodle & $X X$ & $X X X$ & $X X$ & $X X$ & - & - & $X X$ & $x$ \\
\hline Sakai & $X X$ & XXX & $X X$ & $X X$ & - & - & - & - \\
\hline $\begin{array}{l}\text { Redes de } \\
\text { Sensores } \\
\text { (Chang } \\
\text { 2009) }\end{array}$ & $X X$ & $X X$ & $X X X$ & $X X$ & $x$ & $X X X$ & $X X$ & $X X$ \\
\hline $\begin{array}{l}\text { Computaçã } \\
\text { o Ubíqua } \\
\text { (Shih,2011) }\end{array}$ & $x$ & - & $X X$ & $x$ & $X X$ & $X X$ & $X X$ & $X X$ \\
\hline $\begin{array}{l}\text { CPS } \\
\text { (Olympiou, } \\
\text { 2011) }\end{array}$ & $x$ & - & $X X X$ & $x$ & $x$ & $X X X$ & $X X$ & $X X$ \\
\hline $\begin{array}{l}\text { Laboratório } \\
\text { Virtual-Real } \\
\text { (Hoyer, } \\
\text { 2004) }\end{array}$ & $X X$ & - & X & $X X X$ & $x$ & $X X$ & X & X \\
\hline
\end{tabular}

- Não analisado $\quad$ X Ruim

XXX Ótimo

\section{Aspectos Metodológicos}

\subsection{Toogle}

A seguir, é descrita a plataforma Toogle, proposta com o intuito de implementar sistemas físico-virtuais. A plataforma tem por finalidade atender uma série de requisitos listados na seção 5, que estão relacionados ao quadro comparativo apresentado na seção anterior e aos desafios de implantação dos sistemas físico-virtuais. A Figura 2 apresenta a arquitetura da plataforma Toogle.

Middleware e Componentes:

Este módulo é responsável por propiciar a comunicação entre as diferentes entidades físicas e digitais de um ambiente físico-virtual. O módulo atua como uma interface entre os elementos físicos e virtuais e é composto pelo framework ROS (Robot Operating System) [Quigley, 2009], que atua como middleware, fornecendo abstração de hardware, drivers para inúmeros dispositivos e sistema de troca de mensagens, através de um conjunto de ferramentas e bibliotecas.

Toogle Editor:

Esse módulo possibilita operações básicas para edições no ambiente: adicionar, remover e editar componentes. O Toogle Editor se utiliza da ferramenta Blender e ainda possibilita que se edite um conjunto de objetivos associados ao ambiente.

Inteligência do Ambiente:

Este módulo se faz valer do formalismo de descrição de CPS para fornecer um conjunto ordenado de recursos (ações) que conduzirão o ambiente ao alcance dos objetivos.

Toogle Navegador:

O módulo Toogle Navegador possibilita que o usuário interaja com o ambiente. As propriedades dos Objetos Inteligentes e das Entidades Digitais são apresentadas para o usuário através do disparo de sensações multi-modais, como por exemplo, sua volumetria em um cenário 3D, informações sonoras, estereoscópicas, tateis, etc. $\mathrm{O}$ módulo possui ainda um plugin para Internet, permitindo mobilidade na visualização. 


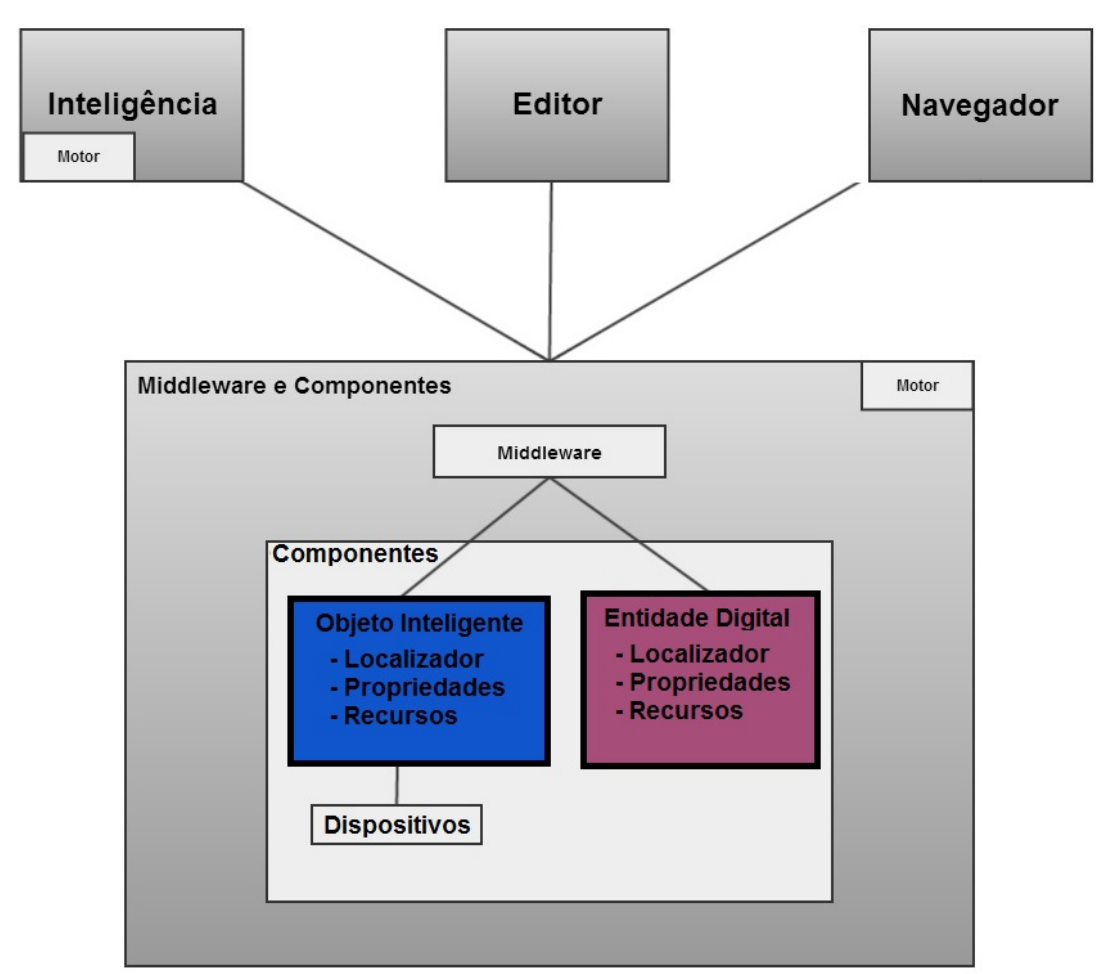

Figura 2: Arquitetura da Plataforma Toogle

\subsection{Ambientes Físico-Virtuais de Aprendizagem (AFVA)}

Pretende-se agora analisar a possibilidade de se integrar elementos reais e virtuais, em ambientes educacionais. Propõe-se que um AFVA seja entendido como um ambiente de aprendizado que possui as sete características de um AVA, apresentadas por Dillenbourg, com o acréscimo de uma oitava, apresentada a seguir: AFVAs devem propiciar que espaços físicos contemplem diversos elementos do mundo virtual. A medida que o aluno se desloque pelos diferentes espaços físicos do seu dia a dia, um AFVA deve permitir que este vivencie espaços educativos durante tais momentos, utilizando-se de elementos reais dos locais e de elementos virtuais. Dessa forma, pretende-se que um AFVA possa prover um alto grau de engajamento do aluno.

Modelo conceitual de um AFVA e sua implementação através da Toogle

A figura 3 apresenta o modelo conceitual adaptado para AFVAs. O modelo também é relacionado com os módulos da plataforma Toogle.

a) Aluno e Contexto: os alunos continuam sendo atores que, para aprender, interagem com o ambiente. O contexto também contribui nos momentos de aprendizagem, podendo inclusive, junto com o aluno, estabelecer novos conteúdos.

b) Professores: são responsáveis pela elaboração dos currículos, pela determinação dos objetivos de aprendizagem e por mediar as adaptações (oportunismo) das situações de aprendizagem disponibilizadas para os alunos. 


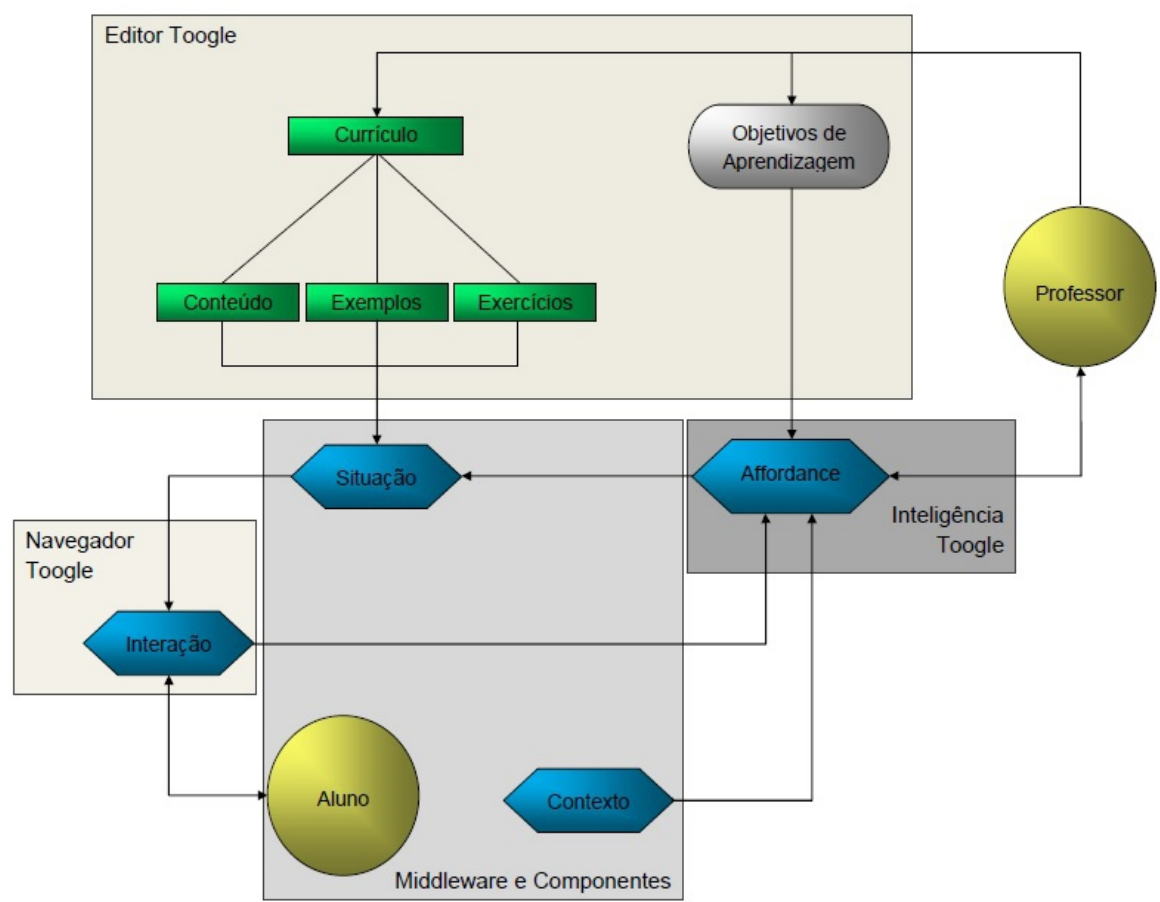

Figura 3: Modelo Conceitual para AFVA (adaptado Dongming Xu, 2005)

c) Situação: semelhante a proposta para os AVAs, a entidade Situação de um AFVA procura formalizar o contexto das situações de aprendizagem através da abordagem STRIPS [Fikes e Nilsson, 1972], e oferecer novas oportunidades para o ensino dos alunos (podem utilizar objetos do mundo físico).

d) Oportunismo: uma vez que o modelo de oportunismo adapta novas situações a partir das interações prévias do aluno, no AFVA, ele deve estar preparado para perceber as novas formas de interação, bem como para sugerir novas situações (com elementos físicos e virtuais).

e) Interação: para um processo de aprendizagem adequado, um AFVA deve focar em proporcionar espaços de interação que permitam a mistura de elementos físicos e virtuais.

f) Objetivos de Aprendizagem: assim como no caso de AVAs, em um AFVA, o professor é responsável pela mediação do processo de aprendizagem e pode indicar o foco das atividades a partir da definição dos objetivos de aprendizagem que envolvam elementos físicos e virtuais.

g) Currículo: o modelo curricular permanece responsável por armazenar as informações do currículo, no entanto, em um AFVA, o professor pode desenvolver uma série de objetos de aprendizagem mais específicos que se utilizem dos elementos físico-virtuais do ambiente.

A partir do modelo conceitual estabelecido, procurou-se relacioná-lo à implementação de um AFVA através da plataforma Toogle. Tal relação é discutida a seguir, a partir da análise dos módulos da Toogle.

a) Editor Toogle: é o ambiente de atuação do professor, onde ele pode criar conteúdos, exercícios, exemplos (inclusive com elementos físico-virtuais) e definir objetivos de 
aprendizagem.

b) Navegador Toogle: é uma ferramenta que permite experiências multimodais, proporcionando interação entre o aluno e as situações que o são apresentadas.

c) Middleware e Componentes: todos componentes existentes no ambiente implementado pela Toogle são classificados como Entidades Digitais (associados aos elementos do espaço virtual) ou Objetos Inteligentes (associados aos elementos do espaço físico). Os contextos dos alunos são formalizados e abstraídos neste módulo como sendo compostos de componentes que contém Localizador, Propriedades e Recurso. As situações de aprendizagem sugeridas aos alunos também são formalizadas pelo módulo Middleware e Componentes.

d) Módulo Inteligência do Ambiente: este módulo está relacionado com o modelo Oportunismo do modelo conceitual. Ele é capaz de, a partir do contexto do aluno, de suas interações e dos objetivos de aprendizagem, planejar uma série de situações possíveis para que se possa chegar a um estado final de aprendizagem almejado.

\section{Resultados}

A seguir, apresenta-se a descrição do desenvolvimento de um AFVA para o estudo de caso descrito abaixo, sob a ótica dos módulos da Toogle e do modelo conceitual proposto para AFVAs:

Um professor pretende criar uma aula sobre determinado assunto, utilizando conteúdos físico-virtuais. Para isso, ele pretende utilizar duas apostilas (arquivos pdf), relacionando-as à localizações geográficas, como bibliotecas, parques, praças, áreas de lazer, etc. Como objetivo de aprendizagem, o professor deseja definir que os alunos recebam e visualizem tais apostilas.

\section{Criação dos componentes através do Editor Toogle}

O desenvolvimento do AFVA teve seu início pela criação dos componentes do ambiente. A figura 4 apresenta um modelo 3D de um prédio com várias salas de aula, que foi inserido como componente inicial para o estudo de caso aqui apresentado.

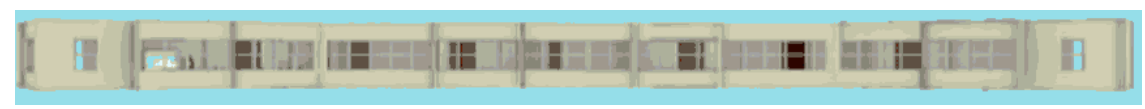

Figura 4: Referencial Inercial do Hiperambiente

Após a inserção do referencial inercial, cada componente do AFVA foi criado, através do Editor Toogle. Cada componente foi associado a uma representação 3D e uma propriedade de posição foi estabelecida. Após, outras propriedades e recursos foram atribuídos aos componentes, de acordo com a necessidade de cada um. A figura 5 apresenta a criação de um componente no hiperambiente. Cabe ressaltar que a propriedade "Localização" do componente Aluno é dinâmica e foi associado ao recurso "Provê_Posição" do componente "Smartphone", fazendo com que a localização do aluno esteja sempre sendo atualizada pelos dados do GPS do smartphone do aluno. Assim, os seguintes componentes foram criados, cada um com suas propriedades e recursos: Sala de aula, Aluno, Professor, Apostila1, Apostila2, Smartphone.

Para o contexto de aprendizado do estudo de caso, associou-se a propriedade "Localização" do componente "Apostila1" a sala 1 do prédio utilizado para os testes, enquanto que a "Localização" do componente "Apostila2" foi associada a sala 2 do mesmo prédio. Para esses dois componentes também foi criado um recurso 
“Enviar_PDF”, que foi escolhido de um banco de recursos implementados e disponíveis no AFVA. Esse recurso, foi implementado para enviar um arquivo pdf a um componente quando as propriedades de localização forem iguais. Para o estudo de caso, quando a propriedade de "Localização" do Aluno for igual a propriedade de "Localização" da "Apostila1", o arquivo pdf, associado ao componente "Apostila1", seja enviado para o Aluno.

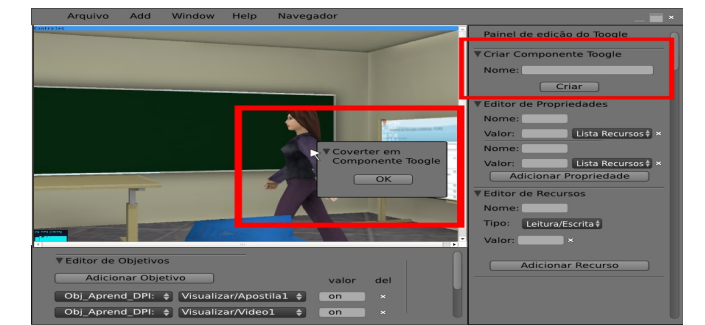

Figura 5: Criação de um componente Toogle

O Editor Toogle possibilita também que os objetivos de aprendizagem sejam definidos, a partir de especificações de algumas propriedades dos componentes do ambiente. Dessa forma, foi estabelecido como objetivos de aprendizagem que as propriedades "Visualizado" dos componentes “Apostila1" e "Apostila2" sejam "verdadeiro". Cabe ressaltas que o recurso "Enviar_PDF" mencionado anteriormente modifica a propriedade "Visualizado" de um componente que recebe o arquivo pdf.

Sob o ponto de vista do modelo conceitual, os componentes “Apostila1” e “Apostila2” são inseridos como conteúdos que fazem parte do modelo currículo. Já os objetivos de aprendizagem definidos no Editor Toogle fazem parte dos objetivos de aprendizagem do modelo conceitual para AFVAs.

Formalização do AFVA (Módulo Middleware e Componentes)

A medida que novos componentes foram inseridos e modificados no Editor Toogle, o módulo Middleware e Componentes se encarregou de abstrair os dispositivos e formalizar o ambiente, gerando um arquivo XML que caracterizou o cenário como um hiperambiente. Esse arquivo XML contém informações dos componentes (Objetos Inteligentes e Entidades Digitais) existentes no ambiente. As propriedades e os recursos dos componentes são descritos em um formato orientado pela abordagem STRIPS e a comunicação dos componentes foi feita através de tópicos e serviços do ROS.

Sob o ponto de vista do modelo conceitual para AFVAs, o módulo Middleware e Componentes pode formalizar tanto o contexto do aluno (sua localização geográfica, por exemplo), quanto as situações de aprendizagem oferecidas (recurso Enviar_PDF).

Adaptação das situações (Módulo Inteligência do Ambiente)

Como já mencionado, o módulo Inteligência do Ambiente, permite que situações sejam planejadas e simuladas, a partir da formalização do mundo em um estado inicial, para se chegar em um estado final de mundo (este elaborado através dos objetivos do ambiente/de aprendizagem). Sendo assim, o estado inicial do ambiente do estudo de caso, após terem sido inseridos os componentes no Editor Toogle, foi recebido pelo módulo Inteligência do Ambiente. O módulo Inteligência do Ambiente também recebeu a formalização do estado futuro do ambiente, gerada a partir dos objetivos de aprendizagem.

Por fim, o módulo Inteligência do Ambiente planejou as ações (execução de recursos) para que o ambiente atingisse o estado futuro, a partir do estado inicial 
(conforme pode ser visto abaixo). Este planejamento foi feito por um motor (engine) presente neste módulo que, constantemente, verifica o estado atual do mundo, planeja os recursos a serem disparados e efetivamente dispara os recursos.

Planejamento: evento (Apostila1,Enviar_PDF), evento (Apostila2,Enviar_PDF)

Este planejamento é associado às adaptações das situações de aprendizagem do modelo de Oportunismo no modelo conceitual proposto. Isto pode ser compreendido no exemplo acima, onde a execução do recurso "Enviar_PDF” é planejado para o Aluno, uma vez que o motor percebe que o objetivo de aprendizagem envolve que a propriedade "Visualizado" seja modificado para "verdadeiro" e consequentemente que o recurso "Enviar_PDF”, se executado, produz tal alteração.

\section{Navegação pelo AFVA (Módulo Toogle Navegador)}

Por fim, após os componentes terem sido criados e formalizados para o estudo de caso, o aluno pôde navegar pelo hiperambiente, através do Navegador Toogle. Todas informações dos componentes são armazenadas e atualizados constantemente em um arquivo XML pelo motor do módulo Middleware e Componentes. Um plug-in, implementado utilizando websocket possibilita que essas informações sejam acessadas em qualquer lugar por um navegador comum. Um cenário, com representações 3D é apresentado de acordo com as propriedades e recursos dos componentes. No estudo de caso aqui apresentado, acessando o navegador o usuário visualiza o prédio inserido como referencial inercial. A medida que o aluno se desloque pelo mundo físico e, através de seu smartphone, seja percebido nas diferentes salas, seu avatar aparece na correspondente sala virtual. Nesse ambiente virtual, os objetos de aprendizagem (Apostila1 e Apostila2) foram representados nas salas correspondentes a suas localizações. A figura 6 ilustra o aluno navegando pela sala de aula físico-virtual e obtendo a visualização do objeto de aprendizagem Apostila1, a medida que ele entra na sala 1 do mundo físico.

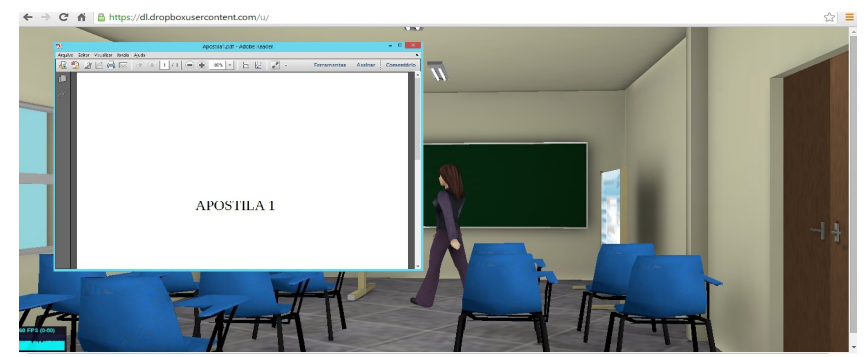

Figura 6: Aluno obtendo visualização de Apostila1 no Navegador Toogle

\section{Discussão}

A fim de discutir sobre o desenvolvimento de um ambiente físico-virtual de aprendizagem através da plataforma Toogle, retoma-se aqui os requisitos definidos para a Toogle e para os AFVAs, analisando-os sob a ótica dos resultados obtidos.

- Informação Multissensorial: o banco de componentes da Toogle inclui objetos de aprendizagem que trabalham com representações 3D, sons, objetos táteis, etc.

- Edição e Navegação: através de Editor Toogle criou-se todo cenário e os componentes existentes do AFVA para o estudo de caso apresentado. Por meio do Navegador Toogle também foi possível a navegação por tal ambiente. 
- Comunicação e interação: toda comunicação e interação entre os componentes do AFVA foi possível através do módulo Middleware e Componentes.

- Inteligência: ainda que de maneira simples, o estudo de caso demonstrou que o módulo Inteligência da Toogle é capaz, a partir da formalização do estado atual do mundo, planejar com algum grau de inteligência, situações a serem oferecidas aos alunos em um AFVA, a fim de que atinjam os objetivos de aprendizagem.

- Engajamento: os níveis de engajamento oferecidos aos alunos parecem ser elevados, uma vez que os diferentes contextos do dia a dia do aluno são levados em conta ao se propor as situações de aprendizagem em um AFVA.

\section{Conclusões}

Este artigo apresentou o conceito de Ambientes Físico-Virtuais de Aprendizagem, que procura integrar ambientes físicos com ambientes virtuais de aprendizagem. A plataforma Toogle foi proposta como implementação de sistemas físico-virtuais. Posteriormente, questões relacionadas a integração dos ambientes físicos e AVAs, foram apresentadas como foco do trabalho, com a proposta de AFVA.

Por fim, os resultados do trabalho se mostraram importantes, uma vez que consolidam uma ferramenta inovadora, que busca contemplar recursos físicos e virtuais para criação de espaços de aprendizagem. A identificação de elementos que constituem esses ambientes físico-virtual de aprendizagem, através de um modelo conceitual, se caracteriza como principal contribuição do trabalho. Em trabalhos futuros, pretende-se aprimorar a plataforma, principalmente quanto a questões referentes ao módulo Inteligência do Ambiente, para que seja possível melhorar os sistemas de percepção e sugestão de situações de aprendizagem. Além disso, novos estudos de casos estão sendo implementados, pretendendo-se assim, ajustar nas próximas etapas do trabalho, tanto a plataforma, quanto o modelo conceitual proposto.

\section{Referências}

Dillenbourg, P. et al. (2002). Virtual learning environments. Proceedings of the 3rd Hellenic Conference'Information \& Communication Technologies in Education.

Dongming, X. et al. (2005). A conceptual model of personalized virtual learning environments. Expert Systems with Applications, 29, 3, 525-534.

Fikes, R. E., e Nilsson, N. J. (1972). STRIPS: A new approach to the application of theorem proving to problem solving. Artificial intelligence, 2(3), 189-208.

Lee, E. A. (2008). Cyber physical systems: Design challenges. 363-369.

Olympiou, G. e Zacharia, Z. C. (2012). Blending physical and virtual manipulatives: An effort to improve students' conceptual understanding through science laboratory experimentation. Science Education, 96, 1, 21-47.

Quigley, M. et al. (2009). ROS: an open-source Robot Operating System. ICRA workshop on open source software. 3,2.

Sha, L., Gopalakrishnan, S., Liu, X. e Wang, Q. (2008). CyberPhysical Systems: A New Frontier. Sensor Networks, Ubiquitous and Trustworthy Computing, 1, 9, 11-13.

Sneha, J. M., e G. S. Nagaraja. (2014). "Virtual Learning Environments-A Survey.". 